\title{
BMJ Open Person-centred rehabilitation: what exactly does it mean? Protocol for a scoping review with thematic analysis towards framing the concept and practice of person-centred rehabilitation
}

\author{
Tiago S Jesus, ${ }^{1}$ Felicity Bright, ${ }^{2}$ Nicola Kayes, ${ }^{2}$ Cheryl A Cott ${ }^{3}$
}

To cite: Jesus TS, Bright F, Kayes $\mathrm{N}$, et al. Personcentred rehabilitation: what exactly does it mean?

Protocol for a scoping review with thematic analysis towards framing the concept and practice of personcentred rehabilitation. BMJ Open 2016;6:e011959. doi:10.1136/bmjopen-2016011959

- Prepublication history for this paper is available online. To view these files please visit the journal online (http://dx.doi.org/10.1136/ bmjopen-2016-011959).

Received 18 March 2016 Revised 21 May 2016 Accepted 30 June 2016

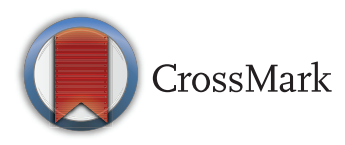

${ }^{1}$ Portuguese Ministry of Education. Aggregation of Schools of Escariz, Escariz, Portugal

${ }^{2}$ Centre for Person Centred Research, School of Clinical Sciences, Auckland University of Technology, Auckland, New Zealand

${ }^{3}$ Faculty of Medicine, Department of Physical Therapy, University of Toronto, Toronto, Ontario, Canada

Correspondence to Dr Tiago S Jesus; jesus-ts@outlook.com

\begin{abstract}
Introduction: Person-centredness is a philosophy for organising and delivering healthcare based on patients needs, preferences and experiences. Although widely endorsed, the concept suffers from a lack of detail and clarification, in turn accounting for ambiguous implementation and outcomes. While a conceptual framework based on a systematic review defines person/patient-centred care components (Scholl et al, 2014), it applies across healthcare contexts and may not be sensitive to the nuances of the rehabilitation of adults with physical impairments. Accordingly, this study aims to build a conceptual framework, based on existing literature, of what person-centredness means in the rehabilitation of adults with physical impairments in the clinical encounter and broader health service delivery.
\end{abstract}

Methods and analysis: We will use a scoping review methodology. Searches on relevant databases will be conducted first, combining keywords for 'rehabilitation', 'person-centered' and associated terms (including patient preferences/experiences). Next, snowball searches (citation tracking, references lists) will be performed. Papers will be included if they fall within predefined selection categories (seen as most likely informative on elements pertaining to personcentred rehabilitation) and are written in English, regardless of design (conceptual, qualitative, quantitative). Two reviewers will independently screen titles and abstracts, followed by screening of the full text to determine inclusion. Experts will then be consulted to identify relevant missing papers. This can include elements other than the peer-reviewed literature (eg, book chapters, policy/legal papers). Finally, information that helps to build the concept and practice of person-centred rehabilitation will be abstracted independently by two reviewers and analysed by inductive thematic analysis to build the conceptual framework.

Dissemination: The resulting framework will aid clarification regarding person-centred rehabilitation, which in turn is expected to conceptually ground and inform its operationalisation (eg, measurement, implementation, improvement). Findings will be

\section{Strengths and limitations of this study}

- A key strength of this study is the use of an increasingly established scoping review approach, followed by an inductive thematic synthesis, to inform the development of a framework of the concept and practice of personcentred rehabilitation.

- Experts will be consulted for identifying missing papers and for potentially modifying the draft conceptual framework and its implications.

- Consistent with the scoping review methodology, quality assessment and the subsequent grading of evidence will not be performed. The study will be limited to papers in the English language.

disseminated through local, national and international stakeholders, both at the clinical and service organisation levels.

\section{INTRODUCTION}

'Person-centredness' refers to a philosophy intended to underpin care and service delivery focused on: meeting the person's needs, values or preferences; optimising the person's experiences with care; and fully involving persons' perspectives into care. These core principles are applicable to the point of care, as well as to the design of healthcare systems, policies and services. ${ }^{1-4}$

Sometimes misunderstood, personcentredness is not just about giving people whatever they want, or simply providing information to them. ${ }^{5}$ It is about interacting with people with dignity, compassion and respect, about seeing the person as an individual and an expert in himself/herself, and about putting people and their families at the centre of decisions (ie, seeking their 
voices, values and circumstances and incorporating these in care decisions). In short, more than a set of activities, person-centredness is a way of thinking and doing things 'with' and 'for' the person, rather than something that is delivered 'to' patients. ${ }^{1}{ }^{5-8}$

Different terms have been interchangeably used to convey these principles; yet, some important nuances may apply in the use of varied terminologies. For example, while the term 'patient-centred' care is often used in the healthcare literature, ${ }^{16-8}$ the term 'personcentredness' has been increasingly preferred since it has a wider, more holistic reach: giving primacy to the 'person' beyond the 'patient'. ${ }^{9}{ }^{10}$ Within that perspective, the unique humane and contextual factors of the person are considered above and beyond the biological factors of the patient who has a disease.

Even though the philosophy of person-centredness seems to be increasingly endorsed across healthcare systems globally, difficulties have arisen when specifically defining and operationalising the approach. This difficulty, in turn, has constrained the implementation of person-centredness into practice. ${ }^{11-16}$ For example, the fuzziness around the meaning of person/patientcentredness means that this ideal is often studied, labelled, measured, implemented or improved in widely varying, sometimes ambiguous ways. ${ }^{10}$ 17-19 This may be one reason why two systematic reviews have found inconsistency regarding the impact of interventions implementing so-called person/patient-centred care approaches. ${ }^{20} 21$

In short, it is unlikely that person-centredness can be optimally attained without improved conceptual clarification. A recent systematic review was performed with the aim of building an integrative framework of patientcentred healthcare. The aims were to depict the elements or dimensions that specifically contribute to, or actually reflect, that concept. ${ }^{22}$ While this review and its results are important, it is unclear whether the broad scope of the model (ie, across healthcare areas) is sensitive to the nuances of specific healthcare fields.

This review focuses on person-centredness in the rehabilitation of people with physical impairments (including cognitive, communication, emotional or neurobehavioural impairments or manifestations associated with or secondary to a physical injury or disease). Such rehabilitation aims to foster the preservation, recovery or compensation of people's functioning, instead of curing any underlying disease. ${ }^{23}$ Accordingly, the meaning and practice of person-centredness may have some unique particularities in rehabilitation contexts. For example, rehabilitation requires active participation in rehabilitation care activities on the part of the individual client, rather than just adherence to medication prescriptions and/or participation in decision-making. ${ }^{24}$ Such participation can be challenged by the presence of cognitive/ communication impairment, which can require particular forms of support from the practitioner. ${ }^{24}$ Clients commonly engage with a multidisciplinary team; enacting person-centred practice may present particular challenges in this context. ${ }^{25}$ Further, family involvement is a common priority within rehabilitation services: at times the 'client' may be the client with the impairment plus their family. ${ }^{23} 24{ }^{26}$ Finally, rehabilitation occurs across the care continuum (eg, in inpatient, outpatient and community settings) and over a long period of time. ${ }^{23}$ So, people's needs may change along the way and require changes in how practitioners and services work, for instance, moving from reducing impairment to compensating for a loss of function or even for the development of any new capacity or function, beyond those actually lost.

All of this indicates that there is a need to focus on the concept and practice of person-centred practice in rehabilitation specifically; a generalist model of person/ patient-centred healthcare may be not applicable to the rehabilitation of people with physical impairments.

Specific terms (eg, client-centred rehabilitation, clientcentred practice) have been used to convey how the concept of person-centredness applies to the rehabilitation paradigm or the involved professions ${ }^{26-28}$-as opposed to what person/patient-centredness would mean in curative-based healthcare areas or professions. ${ }^{26}$ Besides, the ideal of a person-centred rehabilitation is generally endorsed by researchers in the field, 2326 28-31 regardless of the varied terms or nuances in its conceptualisation. Research exploring patient and practitioner perspectives also highlights its importance. ${ }^{28} \quad 32-37$ However, how person-centredness is, or could be, specifically applied in rehabilitation practice is often perceived differently across a range of rehabilitation stakeholders such as patients, providers and other parties. ${ }^{35} 38$

Possibly as a result of that conceptual blurring, or stakeholders' misunderstandings, implementing personcentredness in physical rehabilitation has been problematic. ${ }^{30}{ }^{39-41}$ For instance, while goal setting centred on the person/patient's needs is said to be a component of a person-centredness, it is often absent in rehabilitation goal-setting practices which may focus on provider priorities. ${ }^{38} 4243$ Healthcare systems and structures, professional identities, healthcare teams functioning, external funding and organiational procedures are among the other factors that can also discourage or inadvertently prevent, the delivery of person-centred rehabilitation. ${ }^{26} 363743-45$

Finally, varying research approaches have shown contradictory effects when the philosophy of personcentredness has been applied to rehabilitation. For example, a randomised controlled trial implementing a structured client-centred care approach saw worse outcomes in the intervention arm than in those receiving usual care. ${ }^{46}{ }^{47}$ In contrast, another trial testing a selflabelled 'person-centred' physical therapy intervention (focused on health-enhancing physical activity) found benefits on the main outcome measured (fatigue in persons with rheumatoid arthritis). In addition, a mixedmethods study ${ }^{48}{ }^{49}$ found that the top practitioners (ie, the ones who achieved top patient outcomes-as 
determined by statistical analysis ${ }^{49}$ ) were observed to have a patient/person-centred orientation in their clinical reasoning, an orientation which was not present in practitioners achieving average patient outcomes. ${ }^{48}$

In short, while person-centred rehabilitation seems to be valued in general, its effects and implementation can appear desirable or detrimental depending on how the approach is conceptualised, operationalised or even adapted to the nuances of the rehabilitation of people with physical impairments. While other areas of healthcare have elucidated elements pertaining to the concept, ${ }^{8} 92250$ and some conceptual papers exist in rehabilitation, ${ }^{27-29} 39$ we have found neither a structured review effort nor a resultant framework articulating which elements depict, or best reflect, how the concept and practice of person-centredness specifically applies to the rehabilitation of adults with physical impairments. Therefore, on the basis of the existing literature, the aim of this study is to build a comprehensive framework elucidating both the concept and practice of personcentredness, specifically in the context of the rehabilitation of adults ( $>18$ years) with physical impairments.

\section{METHODS AND ANALYSIS}

We chose a systematic scoping review methodology to map and summarise the published literature related to the concept of person-centred rehabilitation. Following this, we will use an inductive thematic analysis to help build a comprehensive framework that clearly articulates what the concept of person-centredness specifically means in rehabilitation, and how it has been-or might be-translated into actual practice.

While a number of review approaches exist, a scoping review approach was chosen for the following reasons. Scoping review methodology $y^{51-53}$ specifically aims to examine the amount, range and nature of empirical and conceptual research activity in a broad topic area. It systematically maps the body of literature to: identify what is known and not known on a topic (eg, gaps in knowledge and contents covered); guide research agendas; and organise published information on key concepts, theories and evidence reported. ${ }^{51}{ }^{54}$ Inclusively, it can help provide greater conceptual clarity (eg, a conceptual map) about how the literature has addressed a wide and complex topic, ${ }^{55}$ which is consistent with our aims.

Scoping reviews can incorporate theoretical and empirical research, inclusive of a broad range of designs. ${ }^{55}$ Since the aim is to map the existing literature, quality assessment of included articles is not usually performed. If performed, it is not necessarily used to exclude studies from the analysis. ${ }^{51} 56$ Within the context of our review, varying data sources and research designs are equally relevant and appropriate to inform the scoping review results and the resultant conceptual framework.

Alternate review methodologies were considered but were deemed not appropriate for our study aims and purpose. For example, a Cochrane-style 'systematic review' and 'realist review' typically target narrower topics or interventions (ie, either the impact of an intervention or the underlying mechanisms by which it works in varying contexts). We seek conceptual clarification rather than a synthesis of intervention processes and effects. More closely related, the 'meta-narrative review' approach would be a possible alternative (ie, depicting how a complex topic has been historically approached by different research traditions or schools of thought). ${ }^{57-59}$ However, that approach is theoretically driven (eg, starts with a set of theories or conceptual dispositions established a priori, which then help to organise the research reviewed). We start with no a priori map of the literature or conceptions on the meanings or dimensions of person-centred rehabilitation. Scoping reviews are increasingly used as first-time review efforts, that is, on research or conceptual topics that are wide, complex, fragmented or ill-defined. ${ }^{5156}$

Finally, we did not use a specific 'concept analysis'. ${ }^{60} 61$ We are interested in defining both the attributes of concept and the practice (and the translation of the concept to the practice) of person-centred rehabilitation. As such, we are particularly interested in coming to a resultant actionable framework which depicts the elements-and their relationships-which altogether build the ideal, and hopefully also can guide the practice, of 'person-centred rehabilitation'.

In this scoping review study, we use the tenets and specific template of Arksey and O'Malley, ${ }^{53}$ which have been further updated by Levac et al. ${ }^{51}{ }^{52}$ While the review process is typically iterative, the review stages can be organised into the following broad steps: (1) identifying the research question, (2) identifying relevant studies (developing the search), (3) study selection, (4) charting the data (data extraction); (5) collating, summarising and reporting the results and (6) consultation of stakeholders or experts (optional). We depict below how each one is applied to this study.

\section{The review stages}

1. Identifying the research question:

Scoping reviews seek to summarise the breadth of evidence. Consequently, the research question is typically broad. The research question is critical as it provides the overall rationale for decision-making about later study selection and data extraction. After initial engagement with the relevant literature, and while gaining familiarity with the existing body of knowledge, we have developed the following research question: Which elements-both at the service delivery and clinical encounter levelspertain to, and altogether best reflect, both the concept and practice of person-centred rehabilitation when targeting adult populations with physical impairments?

Informed by Morse's discussion on concept analysis, ${ }^{60}$ we use the term 'concept' to refer to the key attributes, preconditions or expected outcomes of person-centred rehabilitation $^{61}$ asking: what does it take to provide 
person-centred rehabilitation and what might result from this? The outputs of this scoping review will elucidate how the attributes of person-centred rehabilitation relate to each other and the surrounding elements (ie, any preconditions, outcomes). The term 'practice' refers to how the underlying concept is, or can be, operationalised and translated into concrete, observable and hopefully replicable behaviours in the practice of the rehabilitation service delivery and clinical practice. In short, as well as clarifying the concept, we aim to present a framework of person-centred rehabilitation that can be actionable in practice.

2. Identifying relevant studies (developing the search)

The search strategy is centred on database searches. Preliminary searches of the MEDLINE database (through PubMed) helped refine the search terms and protocol. Potential search terms and eligibility criteria have been iteratively tested and refined on MEDLINE, by monitoring the total number of entries at the insertion/elimination of any term, and by periodically checking the first 100 references as sorted 'by relevancy'. The final search strategy in MEDLINE combines (ie, using the operator 'AND') alternative keywords related to 'rehabilitation' and 'person-centredness'. In doing this, we have mixed indexed terms (ie, of the Medical Subject Heading (MeSH) tree structure) with nonindexed or free text keywords (including singulars and plurals, and both Britain and American forms-eg, person-centred and person-centered). Using the operator 'NOT', we have eliminated MeSH terms with a rehabilitation focus that are unrelated to the rehabilitation of people with physical impairments (eg, Mouth Rehabilitation, etc). Finally, we narrowed the search to limit the results to adult-related content and articles written in English. That strategy is detailed below.

("Patient-Centered Care"[Mesh] OR "Patient Preference"[Mesh] OR "Patient Experience" OR "Patients Experience" OR "Patient Experiences" OR "Patients Experiences" OR "Patient Perspective" OR "Patients Perspective" OR "Patient Perspectives" OR "Patients Perspectives" OR "person centred" OR "person centered" OR "client centered" OR "client centred") AND ("rehabilitation"[Subheading] OR "rehabilitation"[All Fields] OR "rehabilitation" [MeSH] OR "Physical and Rehabilitation Medicine"[Mesh] OR "Rehabilitation of Speech and Language Disorders"[Mesh] OR "rehabilitation centers"[MeSH] OR "Rehabilitation Nursing"[Mesh] OR "Rehabilitation, Vocational"[Mesh] OR "Activities of Daily Living"[Mesh] NOT "Correction of Hearing Impairment"[Mesh] NOT "Substance Abuse Treatment Centers"[Mesh] NOT "Mouth Rehabilitation"[Mesh]) AND English[lang] AND "adult"[MeSH Terms].

These terms that will be used specifically in the PubMed/MEDLINE search will also guide the searches on other eligible databases (eg, Scopus, CINAHL). It should be noted that the PsychINFO database, which is specific to psychological literature, is not included. In a preliminary search of PsychINFO, we found that the results predominantly pertained to the Rogerian, clientcentred approach to psychotherapy—which is a practice context/healthcare level not related to the scope of this review. As a result, searches on PsychINFO are not likely to be sensitive enough to distinguish papers relevant to our targeted context from the broader literature on client-centred approaches in psychotherapy and counselling. To partly ensure that we do not exclude relevant psychological literature (ie, that which specifically applies to the rehabilitation of people with physical impairments), we will search databases that index journals of health-related psychological content, such as PubMed and Scopus.

Secondary searches will be carried out using 'snowballing' strategies (eg, related citations tracking, manually searching of reference lists of finally selected articles). These are common and effective when searching wide and complex healthcare topics. ${ }^{236263}$ For related citation tracking, and to enhance the consistency and reproducibility of the approach, ${ }^{52}{ }^{56}$ we will follow the first 10-related citations of each article preliminarily selected, and 10 more on each article selected thereafter.

Finally, in the final search stage, we will consult a minimum of five experts in order to identify relevant papers missed by our search. ${ }^{51}$ We will supply those experts with the list of articles preliminarily included, and each of them will be asked to suggest up to 10 more. In this case, experts can recommend literature that is not peer-reviewed (eg, book chapters, policy and legal papers, institutional reports, etc) within relevant inclusion categories (see below). Experts will be identified using a snowballing approach spreading from personal networks of two senior authors who have published widely on person-centred rehabilitation (NK and CC). ${ }^{24} 26323744456465$ The final set of experts, however, must include: recognised scholar(s) and knowledgeable 'insiders' (ie, people living with disability, or their family members, who also have performed roles as disability advocates). All papers, particularly the peerreviewed ones, identified through snowballing strategies or expert consultation will be subject to the inclusion/ exclusion criteria of the study listed below.

3. Study Selection (eligibility criteria)

A variety of literatures are anticipated (eg, with the help of preliminary searches already conducted) to be relevant to this review. This includes conceptual or intervention design papers (eg, person-centred intervention models or related conceptual papers) as well as quantitative (eg, on the measurement of patient's experiences with rehabilitation) and qualitative studies (eg, exploring patient experiences with or perspectives about rehabilitation service delivery and its person-centredness). All of these may further our understanding of person-centred rehabilitation and elucidate its meaning and components. Accordingly, when targeting adult populations, any paper will be eligible for inclusion in this review if it falls into one or more of the following categories:

1. Conceptual, perspective or review papers explicitly (re-)defining or reporting a meaning, framing or dimension(s) relevant to person-centred 
rehabilitation or related concepts (ie, patientcentred, client-centred). The use of the same related terms also applies to the following items;

2. Papers outlining the design or conceptual elements of a novel (self-labelled) approach to a person-centred intervention, addressing either direct patient care or a whole service model.

3. Studies reporting on or analysing the implementation (or lack thereof) of person-centred approaches in rehabilitation.

4. Qualitative studies reporting on the perspectives, experiences or preferences of adults with any physical impairment related to rehabilitation service delivery and its person-centredness.

5. Qualitative studies on providers' perspectives of person-centred rehabilitation or the personcentredness of their care.

6. Quantitative studies on the measurement of personcentred care, patient experiences with service and care or related patients' perspectives-including studies on scale development and psychometric validation.

We believe that data from any of this research might inform elements for a conceptual framework of personcentred rehabilitation.

A priori exclusion criteria are: (1) quantitative and qualitative studies related to or measuring patient 'satisfaction', which is a concept that is expectancy-dependent and increasingly less used (however, studies on the measurement or report of the increasingly used concept of patient 'experience' will be included) ${ }^{66-69}$ (2) studies specifically referring to paediatric populations $(<18$ years) in which the concept would apply differently (eg, given the different legal role of parents); and (3) studies whose contexts primarily addressed refer to psychotherapy and counselling and whose primary/inciting conditions are related to mental health. This, however, does not exclude interventions directly targeting the rehabilitation of any cognitive, emotional and neurobehavioral sequelae of a physical impairment or disease.

After the first scoping exercise, and also depending on the number of papers falling into each inclusion category, a further-that is, a more granular-selection criteria may be applied. For example, single case reports, qualitative studies with a limited number of patient participants, or qualitative studies exclusively reporting providers' perspectives (eg, priority will be given to the patient's voice on what person-centredness is ${ }^{25} 70$ ) could be excluded at a second selection stage. At this stage, we can also exclude studies already synthesised in previous systematic reviews ${ }^{43} 69$ whose specific components are not found likely to add different dimensions towards informing the conceptual framework building.

Finally, we may end up excluding studies with the oldest publication dates-and thereby their extracted content-from the final analytical process towards building the intended framework. It is quite possible that both the concept and the practice of person-centred rehabilitation have evolved over time. If so, we prefer to build the intended framework based on the more contemporary understanding and practices of person-centredness rehabilitation. While this may confound the analysis, the particular instances in which this occurs can be better determined at the analytical stage. As such, we will not exclude references based on publication dates a priori, but may do so within the analytic process. This approach demonstrates a flexible study design. This is consistent with the back-and-forth, iterative nature of typical scoping reviews-given that any alterations to the initial protocol are reported, and that the ability to respond to the main study question is maintained..$^{51-53}$

All the studies primarily included will be reported as part (ie, within the results) of the primary scoping exercise which will map out the existing literature within each inclusion category, even if some are excluded in the second stage of analysis. Additional secondary analytical processes might be triggered by elements or sources excluded from our main analysis (ie, towards building the intended framework). This may well include, for example, an analysis of how the literature, conceptualisation and practices around the ideal of a person-centred rehabilitation have evolved over time.

4. Charting the data (data extraction);

Two authors (TJ and FB) will independently conduct database searches, based on the screening of (1) titles and (2) abstracts, as needed. At this step, any references potentially fitting the inclusion/exclusion criteria will be considered for further analysis. In order to ensure that we capture the breadth of potential studies, full texts will be retrieved and reviewed for eligibility whenever at least one reviewer selected that reference. Both authors (TJ and FB) will independently review each paper selected. When disagreements about eligibility occur, consensus will be reached through discussion with the third and fourth members (NK and CC). Whenever total agreement is not reached, the paper will be included.

A summary of each study's characteristics and findings related to the study's scope (ie, on meanings or dimensions of person-centred rehabilitation) is recorded in data extraction tables together with textual data. These tables will be purposively built by the research team. Data extraction will be carried out independently by two authors (TSJ and FB).

5. Collating, summarising and reporting the results.

First, the findings will incorporate a summative description of the amount and range of the related literature. Then we will present a qualitative framework for both the concept and practice of person-centred rehabilitation, derived from the data extracted from each included study. ${ }^{51}$

Within the first (quantitative) element of results, we will present the number and types of studies included, stratified by the inclusion categories and further organised by other relevant categories (eg, methodologies, publication years). This will provide an overview of the existing research on each matter. A Preferred Reporting Items for Systematic Reviews and Meta-Analyses 
(PRISMA) flow chart will be presented to detail the flow of the process of conducting the review and the references included or eliminated at each step.

The qualitative framework about the concept and practice of person-centred rehabilitation will be constructed in two subsequent stages: (1) define the key attributes, preconditions and likely outcomes within the concept of person-centred rehabilitation, including how all these elements relate to one another; (2) describe how those elements are translated into actual practice by any concrete, potentially replicable behaviours. An inductive 'thematic analysis' is the method used for the analytical process of both those steps.

A thematic analysis ${ }^{71}$ is focused on examining and reporting on patterns (or 'themes') within the data, either semantically present or latent. Thematic analysis involves not just coding, but also interpreting the raw data or primary sources in looking for new, co-occurring or overarching themes and their meanings. Overall, thematic analysis is focused on determining the different themes within a subject and their inter-relationships. These features are important for building the intended framework, given that we are interested in defining which elements pertain to the concept of personcentred rehabilitation, as well as in how they relate to each other, and finally how they can be translated into practice. ${ }^{72}$ Our thematic analysis will be inductive in nature. While principles ${ }^{73}$ and elements ${ }^{22}$ of person/ patient-centredness exist for broad healthcare (and we may be partly influenced by knowing them), our intent is to build a comprehensive framework that reflects the concept and practice of person-centredness in rehabilitation from the point of view of its specific literature.

Methodologically, the thematic analysis will be performed accordingly to Braun and Clarke's six-phase guide: $^{71}$ (1) Familiarisation with the data; (2) Generating initial codes; (3) Searching for themes; (4) Reviewing themes; (5) Defining and naming themes; and (6) Producing the report. The first elements of this analysis (ie, until step four, inclusively) will be carried out by two investigators (TSJ and FB) independently. All the authors will then actively and iteratively contribute to 'reviewing of themes' and in performing the remaining steps.

Overall, the core themes identified through the analysis will serve as elements of the comprehensive framework on the concept and practice of person-centred rehabilitation. A draft framework will be only considered complete by the authors once the categories/elements are organised and visually displayed in (inter-)relation to each other into a coherent framework of person-centred rehabilitation that embeds the elements of both its concept and practice.

\section{Experts' consultation}

The consultation of experts is an optional but recommended step in scoping reviews. ${ }^{51}$ The goals can vary from shaping the scope of the review, helping with finding relevant articles (as carried out for this review: see the section 'identifying relevant studies'), and also reinterpreting the review results and its implications-as we aim to do as well. Their feedback may inform additional analyses. This may include getting back to the raw data and its thematic interpretation and/or a redefinition or rearrangement of themes into the conceptual framework itself. Alongside, draft implications of the framework will also be provided to the experts in order to be challenged by them, which may also trigger further revisions. A maximum of two iterations with experts may occur. In a previous stage within this protocol (ie, identification of relevant studies), we have mentioned that we will engage a set of experts in detecting any relevant papers. Those same experts will then be also invited to participate later in in this process towards any rearrangement of the draft framework. All alterations to the draft framework, resulting from the experts' input, will be explicitly reported.

\section{ETHICS AND DISSEMINATION}

The aim of this project is to produce a conceptual framework of person-centredness for rehabilitation of adults with physical impairments, based on a scoping review of the rehabilitation-specific literature.

We anticipate that the research will provide several key outcomes such as an improved clarification, and a more common understanding, of the meaning of personcentred rehabilitation and of any of its components. The robust model developed is anticipated to provide a shared conceptual basis and a model for practice informing further work exploring how best to operationalise, implement, measure and improve personcentredness in rehabilitation at both the clinical encounter and service-design level.

The review and conceptual framework will be published in an international interdisciplinary rehabilitation journal in order to reach an interdisciplinary, multistakeholder rehabilitation audience. Additionally, the study will be presented at one of the largest rehabilitation or health improvement conferences (eg, American Congress of Rehabilitation Medicine, Australasian Society for the Study of Brain Impairment), which can be another means to reach this intended audience. To disseminate findings for other key stakeholders (ie, at the broader health system level), presentations can also be made at relevant conferences (eg, the APAC Forum, an Asia-Pacific conference on healthcare transformation). Moreover, the work can also be disseminated though local agencies very directly interested in the matter (eg, the Accident Compensation Corporation in New Zealand: the local fund for accident-related rehabilitation). Finally, we intend to link with relevant worldwide recognised organisations such as Kings Fund and the Health Foundation (both based in the UK), the Institute for Healthcare Improvement and the Institute for Patient-Centered and Family-Centered Healthcare (both in the USA). The goal is to make the framework 
resulting from this review available on these organisations' websites, which are accessed by a vast and nearly global interested audience.

Contributors TSJ provided general guidance to the research team and was involved in study conception, preliminary literature review, content expert input, design of the search strategy, writing of the protocol, scoping review frameworks and analysis. FB was involved in writing and editing of the protocol and content expert input. NK was involved in editing of the protocol and content expert input. CAC was involved in study conception, editing of the protocol and content expert input. All authors have made substantive intellectual contributions to the development of this protocol. All authors read and approved the manuscript.

Competing interests None declared.

Provenance and peer review Not commissioned; externally peer reviewed.

Open Access This is an Open Access article distributed in accordance with the Creative Commons Attribution Non Commercial (CC BY-NC 4.0) license, which permits others to distribute, remix, adapt, build upon this work noncommercially, and license their derivative works on different terms, provided the original work is properly cited and the use is non-commercial. See: http:// creativecommons.org/licenses/by-nc/4.0/

\section{REFERENCES}

1. Berwick DM. A user's manual for the IOM's 'Quality Chasm' report. Health Aff (Millwood) 2002;21:80-90.

2. Berwick DM. What 'patient-centered' should mean: confessions of an extremist. Health Aff (Millwood) 2009;28:w555-65.

3. Entwistle VA, Watt IS. Treating patients as persons: a capabilities approach to support delivery of person-centered care. Am J Bioeth 2013;13:29-39.

4. Duggan PS, Geller G, Cooper LA, et al. The moral nature of patient-centeredness: is it "just the right thing to do"? Patient Educ Couns 2006;62:271-6.

5. Collins A. Measuring what really matters: towards a coherent measurement system to support person-centred care. London Health Foundation, 2014

6. Institute of Medicine. Crossing the quality chasm. Washington DC: National Academies Press, 2001.

7. Gerteis M, Edgman-Levitan S, Daley J, et al. Through the patient's eyes: understanding and promoting patient-centered care. San Francisco, CA: Jossey-Bass, 1993.

8. Mead N, Bower P. Patient-centredness: a conceptual framework and review of the empirical literature. Soc Sci Med 2000;51:1087-110.

9. Morgan S, Yoder LH. A concept analysis of person-centered care. $J$ Holist Nurs 2012;30:6-15.

10. Silva D. Helping measure person-centred care: a review of evidence about commonly used approaches and tools used to help measure person-centred care. London: Health Foundation, 2014.

11. Lawrence M, Kinn S. Defining and measuring patient-centred care: an example from a mixed-methods systematic review of the stroke literature. Health Expect 2012;15:295-326.

12. Rademakers J, Delnoij D, Nijman J, et al. Educational inequalities in patient-centred care: patients' preferences and experiences. BMC Health Serv Res 2012;12:261.

13. Chewning B, Bylund CL, Shah B, et al. Patient preferences for shared decisions: a systematic review. Patient Educ Couns 2012;86:9-18.

14. Scholl I, Zill JM, Härter M, et al. How do health services researchers understand the concept of patient-centeredness? Results from an expert survey. Patient Prefer Adherence 2014;8:1153-60.

15. Gillespie R, Florin D, Gillam $S$. How is patient-centred care understood by the clinical, managerial and lay stakeholders responsible for promoting this agenda? Health Expect 2004;7:142-8.

16. Olsson LE, Jakobsson Ung E, Swedberg K, et al. Efficacy of person-centred care as an intervention in controlled trials-a systematic review. J Clin Nurs 2013;22:456-65.

17. Epstein RM, Franks $\mathrm{P}$, Fiscella $\mathrm{K}$, et al. Measuring patient-centered communication in patient-physician consultations: theoretical and practical issues. Soc Sci Med 2005;61:1516-28.

18. Mead N, Bower P. Measuring patient-centredness: a comparison of three observation-based instruments. Patient Educ Couns 2000;39:71-80
19. Smith RC, Dwamena FC, Grover M, et al. Behaviorally defined patient-centered communication-a narrative review of the literature. $J$ Gen Intern Med 2011;26:185-91.

20. Dwamena F, Holmes-Rovner M, Gaulden CM, et al. Interventions for providers to promote a patient-centred approach in clinical consultations. Cochrane Database Syst Rev 2012;12:CD003267.

21. Lewin SA, Skea ZC, Entwistle V, et al. Interventions for providers to promote a patient-centred approach in clinical consultations. Cochrane Database Syst Rev 2001;(4):CD003267.

22. Scholl I, Zill JM, Härter M, et al. An integrative model of patient-centeredness-a systematic review and concept analysis. PLOS ONE 2014;9:e107828.

23. Jesus TS, Hoenig $\mathrm{H}$. Post-acute rehabilitation quality of care: toward a shared conceptual framework. Arch Phys Med Rehabil 2015;96:960-9.

24. Bright FA, Kayes NM, Worrall L, et al. A conceptual review of engagement in healthcare and rehabilitation. Disabil Rehabil 2015;37:643-54.

25. Sumsion T, Lenchucha R. Therapists' perceptions of how teamwork influences client-centred practice. Br J Occup Ther 2009;72:48-54

26. Cott CA. Client-centred rehabilitation: client perspectives. Disabil Rehabil 2004;26:1411-12.

27. Mroz TM, Pitonyak JS, Fogelberg D, et al. Client centeredness and health reform: key issues for occupational therapy. Am J Occup Ther 2015;69:1-8.

28. Sumsion T, Law M. A review of evidence on the conceptual elements informing client-centred practice. Can J Occup Ther 2006:73:153-62.

29. Hunt MR, Ells C. A patient-centered care ethics analysis model for rehabilitation. Am J Phys Med Rehabil 2013;92:818-27.

30. Gzil F, Lefeve C, Cammelli M, et al. Why is rehabilitation not yet fully person-centred and should it be more person-centred? Disabil Rehabil 2007;29:1616-24.

31. Kramer AM. Reabilitation care and outcomes from the patient's perspective. Med Care 1997;35(6 Suppl):JS48-57; discussion JS58-63.

32. Fadyl JK, McPherson KM, Kayes NM. Perspectives on quality of care for people who experience disability. BMJ Qual Saf 2011;20:87-95.

33. Van de Velde D, Devisch I, De Vriendt P. The client-centred approach as experienced by male neurological rehabilitation clients in occupational therapy. A qualitative study based on a grounded theory tradition. Disabil Rehabil 2016;38:1567-77.

34. D'Cruz K, Howie L, Lentin P. Client-centred practice: perspectives of persons with a traumatic brain injury. Scand J Occup Ther 2016;23:30-8.

35. Maitra KK, Erway F. Perception of client-centered practice in occupational therapists and their clients. Am J Occup Ther 2006:60:298-310.

36. Levack WM, Dean SG, Siegert RJ, et al. Navigating patient-centered goal setting in inpatient stroke rehabilitation: how clinicians control the process to meet perceived professional responsibilities. Patient Educ Couns 2011;85:206-13.

37. Bright FA, Boland P, Rutherford SJ, et al. Implementing a client-centred approach in rehabilitation: an autoethnography. Disabi Rehabil 2012;34:997-1004.

38. Sugavanam T, Mead G, Bulley C, et al. The effects and experiences of goal setting in stroke rehabilitation-a systematic review. Disabil Rehabil 2013;35:177-90.

39. Leplege A, Gzil F, Cammelli M, et al. Person-centredness: conceptual and historical perspectives. Disabil Rehabil 2007;29:1555-65.

40. McPherson KM, Siegert RJ. Person-centred rehabilitation: rhetoric or reality? Disabil Rehabil 2007;29:1551-4

41. Hammell KR. Client-centred practice in occupational therapy: critical reflections. Scand J Occup Ther 2013;20:174-81.

42. Rosewilliam S, Sintler C, Pandyan AD, et al. Is the practice of goal-setting for patients in acute stroke care patient-centred and what factors influence this? A qualitative study. Clin Rehabil 2016:30:508-19.

43. Rosewilliam S, Roskell CA, Pandyan AD. A systematic review and synthesis of the quantitative and qualitative evidence behind patient-centred goal setting in stroke rehabilitation. Clin Rehabil 2011;25:501-14.

44. Mudge S, Stretton C, Kayes N. Are physiotherapists comfortable with person-centred practice? An autoethnographic insight. Disabil Rehabil 2014;36:457-63.

45. Papadimitriou C, Cott C. Client-centred practices and work in inpatient rehabilitation teams: results from four case studies. Disabil Rehabil 2015;37:1135-43.

46. Eyssen IC, Steultjens MP, de Groot V, et al. A cluster randomised controlled trial on the efficacy of client-centred occupational therapy 
in multiple sclerosis: good process, poor outcome. Disabil Rehabil 2013;35:1636-46.

47. Eyssen IC, Dekker J, de Groot V, et al. Client-centred therapy in multiple sclerosis: more intensive diagnostic evaluation and less intensive treatment. J Rehabil Med 2014;46:527-31.

48. Resnik L, Jensen GM. Using clinical outcomes to explore the theory of expert practice in physical therapy. Phys Ther 2003;83:1090-106.

49. Resnik L, Hart DL. Using clinical outcomes to identify expert physical therapists. Phys Ther 2003;83:990-1002.

50. Constand MK, MacDermid JC, Dal Bello-Haas V, et al. Scoping review of patient-centered care approaches in healthcare. BMC Health Serv Res 2014;14:271.

51. Levac D, Colquhoun H, O'Brien KK. Scoping studies: advancing the methodology. Implement Sci 2010;5:69.

52. Colquhoun $\mathrm{HL}$, Levac D, O'Brien KK, et al. Scoping reviews: time for clarity in definition, methods, and reporting. J Clin Epidemiol 2014;67:1291-4.

53. Arksey H, O'Malley L. Scoping studies: towards a methodological framework. Int J Soc Res Methodol 2005;8:19-32.

54. Anderson S, Allen P, Peckham S, et al. Asking the right questions: scoping studies in the commissioning of research on the organisation and delivery of health services. Health Res Policy Syst 2008;6:7.

55. Davis K, Drey N, Gould D. What are scoping studies? A review of the nursing literature. Int J Nurs Stud 2009;46:1386-400.

56. Pham MT, Rajic A, Greig JD, et al. A scoping review of scoping reviews: advancing the approach and enhancing the consistency. Res Synth Methods 2014;5:371-85.

57. Greenhalgh T, Robert G, Macfarlane F, et al. Diffusions of innovations in service organizations: systematic review and recommnedations. Milbank Q. 2004;82:581-629.

58. Greenhalgh T, Robert G, Macfarlane F, et al. Storylines of research in diffusion of innovation: a meta-narrative approach to systematic review. Soc Sci Med 2005;61:417-30.

59. Wong G, Greenhalgh T, Westhorp G, et al. RAMESES publication standards: meta-narrative reviews. BMC Med 2013;11:20.

60. Morse JM. Exploring the theoretical basis of nursing using advanced techniques of concept analysis. Adv Nurs Sci 1995;17:31-46.
61. Morse JM, Mitcham C, Hupcey JE, et al. Criteria for concept evaluation. J Adv Nurs 1996;24:385-90.

62. Jesus TS, Silva IL. Toward an evidence-based provider-patient communication in rehabilitation: pathways linking communication elements to better rehabilitation outcomes. Clin Rehabil 2016;30:315-28.

63. Greenhalgh T, Peacock R. Effectiveness and efficiency of search methods in systematic reviews of complex evidence: audit of primary sources. BMJ 2005;331:1064-5.

64. Levack WM, Boland P, Taylor WJ, et al. Establishing a person-centred framework of self-identity after traumatic brain injury: a grounded theory study to inform measure development. BMJ Open 2014:4:e004630.

65. Miller D, Steele Gray C, Kuluski K, et al. Patient-centered care and patient-reported measures: let's look before we leap. Patient 2015;8:293-9.

66. Black N, Jenkinson C. Measuring patients' experiences and outcomes. BMJ 2009;339:b2495.

67. Medina-Mirapeix F, Jimeno-Serrano FJ, Escolar-Reina $P$, et al. Is patient satisfaction and perceived service quality with musculoskeletal rehabilitation determined by patient experiences? Clin Rehabil 2013;27:555-64

68. Medina-Mirapeix F, Del Baño-Aledo ME, Martínez-Payá JJ, et al. Development and validity of the questionnaire of patients experiences in postacute outpatient physical therapy settings. Phys Ther 2015;95:767-77.

69. McMurray J, McNeil H, Lafortune $\mathrm{C}$, et al. Measuring patients' experience of rehabilitation services across the care continuum. Part II: key dimensions. Arch Phys Med Rehabil 2016;97: 121-30.

70. Bensing J, Rimondini M, Visser A. What patients want. Patient Educ Couns 2013;90:287-90.

71. Braun V, Victoria C. Using thematic analysis in psychology. Qual Res Psychol 2006;3:83.

72. Hsieh HF, Shannon SE. Three approaches to qualitative content analysis. Qual Health Res 2005;15:1277-88.

73. Picker Institute. Principles of patient-centered care. [Online]. http:// pickerinstitute.org/about/picker-principles/. (accessed 12 Dec 2015). 